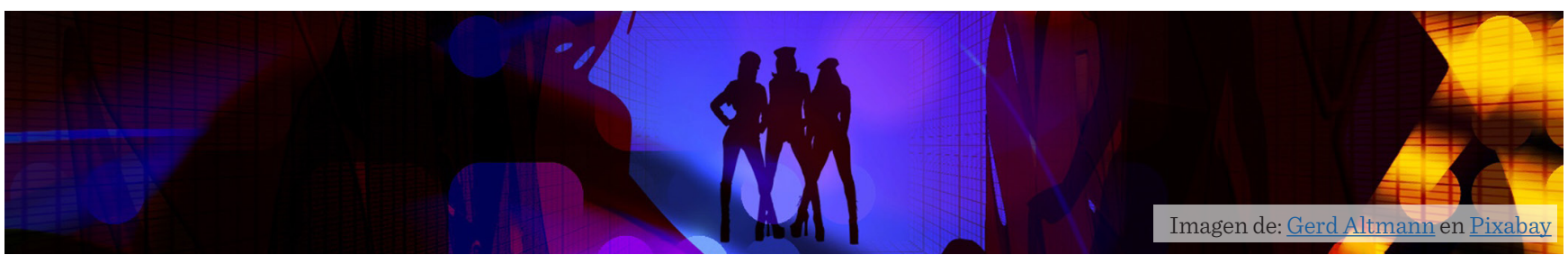

\title{
Mecanismos de reclutamiento utilizados por las redes de tráfico sexual en Nevada, Estados Unidos
}

\section{Recruiting mechanisms used by sex trafficking networks in Nevada, United States}

Karla Lorena Andrade-Rubio*, Simón Pedro Izcara-Palacios

\section{RESUMEN}

El comercio sexual de jóvenes, que son llevadas desde Centroamérica y México hacia Estados Unidos, ha sido ampliamente documentado, y sin embargo, sigue siendo un problema grave, del que se desconocen sus cambiantes dinámicas y estrategias de reclutamiento, ya sean voluntarias o forzadas. El objetivo de este trabajo fue examinar los mecanismos utilizados por las redes de traficantes para reclutar mujeres migrantes, para ser prostituidas en $\mathrm{Ne}-$ vada, Estados Unidos. Este trabajo está fundamentado en una metodología cualitativa, que incluye entrevistas a 3 reclutadores centroamericanos y 12 mujeres de México y Centroamérica. Los principales resultados encontrados indican que estas redes reclutan preferentemente a menores de edad, vulnerables, con un pasado relacionado con la prostitución y necesidades económicas apremiantes. Los mecanismos de reclutamiento utilizados por estas redes no se basan en el uso de la violencia, sino en el abuso de una situación de vulnerabilidad. En conclusión, el hecho de no recurrir a estrategias de reclutamiento violentas disminuye el riesgo de esta actividad, ya que las mujeres no les denuncian, porque no se consideran víctimas.

PALABRAS CLAVE: prostitución, trata, mujeres migrantes, redes de traficantes, Nevada.

\section{ABSTRACT}

The international sex trade, involving young people who are taken from Central America and Mexico to the United States, has been widely documented. However, it remains a serious problem, from which its changing dynamics and recruitment strategies, whether voluntary or forced, are unknown. The objective of this paper was to examine the mechanisms used by sex smuggling networks to recruit migrant women to be prostituted in Nevada, USA. This research is based on a qualitative methodology that includes interviews with 3 Central American recruiters and 12 women from Mexico and Central America. The main results indicate that these networks recruit predominantly vulnerable underage girls with a past associated with prostitution and towering economic necessities. Recruitment mechanisms used by these networks are not based on the use of violence, but on the abuse of a position of vulnerability. In conclusion, not resorting to violent recruitment strategies reduces the risk of this activity, since women do not report them because they do not consider themselves victims.

KEYWORDS: prostitution, sex trafficking, migrant women, sex smuggling networks, Nevada.

*Correspondencia: kandrade@uat.edu.mx/Fecha de recepción: 18 de julio de 2019/Fecha de aceptación: 20 de marzo 2020/Fecha de publicación: 31 de julio de 2020.

Universidad Autónoma de Tamaulipas, Unidad Académica de Trabajo Social y Ciencias para el desarrollo Humano, Centro Universitario Victoria, Ciudad Victoria, Tamaulipas, México, C.P. 87149. 


\section{INTRODUCCIÓN}

i La reflexión del pensamiento feminista sobre el proxenetismo, el trabajo sexual y la trata de personas, con fines de explotación sexual, está fundamentada en diferentes interpretaciones del concepto filosófico de libertad (Cacho, 2010: 172). El feminismo abolicionista antiprostitución parte del concepto rousseauniano de libertad (Cobo, 2017: 170). Para Rousseau (1999: 57), renunciar a la libertad es incompatible con la naturaleza humana. Es absurdo, inconcebible, ilegítimo y nulo; aunque redunde en provecho propio. De aquí se deduce que una mujer no puede consentir en prostituirse, porque esto equivaldría a intercambiar su libertad por un beneficio económico. Según este pensamiento, el transporte de mujeres para la prostitución constituye trata (Jeffreys, 2010; Warren, 2012; Szasz, 2013; Banyard, 2016; Cobo, 2017; Shapiro y Hughes, 2017; Farley, 2018; Moran y Farley, 2019). El ejemplo paradigmático de esta línea de argumentación aparece reflejado en el código penal brasileño, modificado en 2005 y 2009, que define como trata, con fines de explotación sexual, toda forma de facilitación de la entrada o salida del país para trabajar en la prostitución (Blanchette y Da-Silva, 2012: 113).

Como contraste, el feminismo pro-prostitución parte del concepto kantiano de libertad. Para Kant (2017), la libertad es innata al hombre. El origen de su conducta está en sí mismo, de manera que su voluntad no puede estar determinada de modo heterónomo por causas ajenas. De aquí se deriva la capacidad para consentir, de una mujer, su prostitución. Según este argumento, el transporte de mujeres para la prostitución no constituye trata si son conducidas con su consentimiento (Pheterson, 2000; Agustín, 2007; Doezema, 2010; Hua, 2011; Brents, 2016). El ejemplo paradigmático de esta línea de argumentación se encuentra en la legislación turca que, tras la reforma de 2005, define como trata, con fines de explotación sexual, la facilitación de la entrada de una persona extranjera para ser forzada a prostituirse sin percibir una remuneración económica (Coskun, 2016: 446), de mo- do que cualquier otra situación es entendida como tráfico y no como trata (Cacho, 2010: 2346).

Los protocolos complementarios de la Convención de Naciones Unidas contra el Crimen Organizado Transnacional, del año 2000, reflejan esta dicotomía. Los protocolos establecen una distinción entre "tráfico" y "trata". Mientras el tráfico es un crimen contra el Estado, la trata es un crimen contra las personas (Izcara-Palacios, 2019a; 2020). En ningún apartado del protocolo de Naciones Unidas para prevenir, reprimir y sancionar la trata, o protocolo de Palermo, se define como trata la participación voluntaria, consentida, de adultos mayores de edad, en el comercio sexual (Lepp, 2002: 92). Es decir, el tráfico implica consentimiento, mientras que la trata comporta ausencia de consentimiento, aunque no se tendrá en cuenta el consentimiento de toda persona menor de 18 años. Sin embargo, la relación dialéctica entre los conceptos de "consentimiento", impulsado por el feminismo proprostitución, y "vulnerabilidad", impulsado por el feminismo antiprostitución, impide delimitar de modo preciso la barrera que separa estos conceptos.

Es a través de la inclusión de elementos contradictorios, como pudo llegarse a la posición de consenso plasmada en el protocolo de $\mathrm{Pa}-$ lermo. Como consecuencia, desde diferentes posicionamientos políticos y teóricos, el protocolo de Palermo es interpretado de modo diferente (Andrade-Rubio e Izcara-Palacios, 2019: 4). La postura neo-abolicionista establece una equivalencia entre prostitución y trata con fines de explotación sexual (Montiel-Torres, 2018: 49), define el tráfico de mujeres para la prostitución como trata (Farley, 2013; Szasz, 2013: 87; Orozco, 2015: 71; Banyard, 2016: 67; Moran y Farley, 2019) y promueve la imposición de controles migratorios más estrictos para combatir el tráfico de mujeres para el comercio sexual (Warren, 2012: 115; Cobo, 2017: 152 ). Por otra parte, el feminismo defensor del comercio sexual busca que no se utilice el estigma de la prostitución para poner barreras a la migra- 
ción femenina (Doezema, 2000: 40; Pheterson, 2000: 27) y subraya que el tráfico sexual no incluye siempre engaño y coacción (Pheterson, 2000: 128), sino que muchas mujeres de países en desarrollo buscan emigrar a países desarrollados para trabajar en el comercio sexual (Agustín, 2007 ; Hua, 2011; Blanchette y Da-Silva, 2012).

La legislación estadounidense tipifica toda forma de tráfico de mujeres para la prostitución como trata, pero los únicos delitos punibles son las formas severas de trata, y el tráfico de mujeres mayores de edad sin coacción no lo es (Izcara-Palacios y Andrade-Rubio, 2016; 2018). La legislación mexicana es más severa (Orozco, 2015: 16; Correa-Cabrera y SandersMontandon, 2018), ya que subsume la noción de tráfico en el concepto de trata, debido a que en el artículo 10 de la ley general para prevenir, sancionar y erradicar los delitos en materia de trata de personas de 2012, se elimina la ausencia de consentimiento en la definición de la trata, y en el artículo 40 se subraya explícitamente que el consentimiento, cualquiera que sea la edad de la persona, es irrelevante. La legislación de los países centroamericanos presenta muchas similitudes con la mexicana, aunque Nicaragua es el país que tipifica la trata de modo más extenso (Izcara-Palacios, 2019b).

En Estados Unidos, la prostitución está prohibida. Los proxenetas y propietarios de burdeles enfrentan las mayores penas, pero 9 de cada 10 arrestos recaen sobre las mujeres en prostitución (Villacampa-Estiarte, 2012: 96). El único estado donde la prostitución está legalizada es Nevada (Skilbrei, 2019; Walks, 2019). Esto obedece a la dependencia de la economía de este estado de actividades como el juego o el turismo. Esto ha hecho que en las áreas rurales, no así en las urbanas, el comercio sexual sea legal (Bingham, 1998: 85; Brents, 2016). Nevada se caracteriza por una cultura hipersexualizada (Brents, 2016; Jackson y col., 2018), donde la publicitación de la mercantilización del sexo es omnipresente en áreas donde la prostitución está prohibida
(Wagner y col., 2016: 2). La industria del sexo de Nevada tiene una faceta legal (burdeles en condados rurales, bares, clubes nudistas, entre otros), que emplea principalmente a mujeres autóctonas. Sin embargo, los modos predominantes de prostitución se desarrollan al margen de la legalidad. La prostitución ilegal no se reduce al comercio sexual callejero, sino que también abarca a escorts independientes, casinos, bares, clubs, casas de citas, hoteles, o agencias de entretenimiento adulto (Macfarlane y col., 2017: 13). Esta faceta ilegal se nutre en gran medida con la llegada de jóvenes latinoamericanas y centroamericanas para ser empleadas en la prostitución en $\mathrm{Ne}$ vada. En el presente estudio, se denomina redes de traficantes a los grupos de personas que se dedican al reclutamiento y transporte de mujeres, desde su país de origen hasta el lugar de destino (Andrade-Rubio e Izcara-Palacios, 2019).

La prostitución legal en Nevada ha sido estudiada de modo exhaustivo (Brents y Hausbeck, 2007; Brents y col., 2010; Seals, 2015; Brents, 2016; Raphael, 2018; Blithe y col., 2019; Walks, 2019), así como la prostitución ilegal autóctona (Farley, 2007; Wagner y col., 2016; Macfarlane y col., 2017). Por otra parte, son numerosas las investigaciones realizadas en México sobre las técnicas de neutralización utilizadas para minimizar la responsabilidad de quienes trafican mujeres para la prostitución. Estos estudios concluyen que las mujeres son inducidas a la prostitución a través de elaborados procesos, que incluyen elementos que van del enamoramiento al aislamiento social, y tienen como objeto anular la autonomía de las primeras (Cacho, 2010; González-Báez, 2014; Montiel-Torres, 2015; Andrade-Rubio, 2016; Luna-Alfaro, 2016; Montiel-Torres, 2018). Mientras los estudios realizados en México aparecen centrados en los mecanismos de reclutamiento de mujeres autóctonas para el mercado nacional, los estudios realizados en Estados Unidos sobre la prostitución ilegal en Nevada aparecen centrados principalmente en las poblaciones autóctonas blanca y afroamericana (Wagner y col., 2016: 16). Por lo 
tanto, no existe un corpus académico que examine los mecanismos, a través de los cuales, las redes de tráfico sexual conducen a mujeres foráneas para saciar la demanda del mercado hipersexualizado de Nevada.

Este artículo tuvo como objetivo examinar cuáles son los mecanismos de reclutamiento utilizados por las redes de traficantes que conducen mujeres de México y Centroamérica para ser prostituidas en Nevada.

\section{MATERIALES Y MÉTODOS}

Para la realización de esta investigación se utilizó un enfoque metodológico cualitativo. La técnica que se utilizó para recabar la información fue la entrevista en profundidad, y los participantes fueron seleccionados a través del muestreo en cadena. Los y las entrevistados/as fueron contactados a partir de informantes que promovieron el contacto, tanto con mujeres migrantes prostituidas en Nevada, como con reclutadores. Las entrevistas fueron realizadas en espacios públicos, tanto abiertos como cerrados. Las entrevistas fueron grabadas y transcritas de modo literal. Se entrevistaron mujeres y reclutadores (Tablas 1 y 2); y el trabajo de campo se realizó en México y en Estados Unidos.
Por una parte, fueron entrevistadas 12 mujeres de México y Centroamérica conducidas hasta Nevada por redes de traficantes; 5 se encontraban en tránsito por México, ya que fueron deportadas de Nevada, y 7 residían en Nevada.

Las mujeres en tránsito fueron entrevistadas entre 2012 y 2016 en diferentes áreas de México (Ciudad de México, Tamaulipas, Veracruz y Coahuila). Ellas habían permanecido en $\mathrm{Ne}-$ vada algo más de 9 años, hasta que finalmente fueron deportadas hasta sus países de origen. Después de permanecer unos meses con sus familias decidieron retornar al norte; 4 lo hicieron con la ayuda de un patrón de $\mathrm{Ne}$ vada, que había contratado a una red de traficantes para conducirlas a Estados Unidos; 2 de las entrevistadas eran originarias de El Salvador, 2 procedían de Honduras y 1 era de Guatemala. Las edades de las 5 entrevistadas oscilaban entre los 20 y los 30 años. Comenzaron a trabajar a una edad muy temprana para apoyar la economía familiar, principalmente en actividades domésticas, comercio ambulante y agricultura, y su nivel educativo era casi nulo (Tabla 3).

Las mujeres entrevistadas en Nevada, habían residido en ese estado durante una media de 5.4

- Tabla 1. Descripción de las mujeres entrevistadas.

Table 1. Description of interviewed women.

\begin{tabular}{|c|c|c|c|c|}
\hline Entrevistada & Edad (años) & & tio de entrevista & Fecha \\
\hline Adela & 30 & \multirow{5}{*}{ 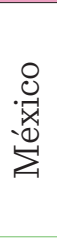 } & Ciudad de México & septiembre de 2012 \\
\hline Amalia & 28 & & Veracruz & julio de 2014 \\
\hline Bruna & 26 & & Veracruz & julio de 2014 \\
\hline Engracia & 20 & & Coahuila & marzo de 2015 \\
\hline Eugenia & 25 & & Tamaulipas & julio de 2016 \\
\hline Gabriela & 22 & \multirow{7}{*}{ 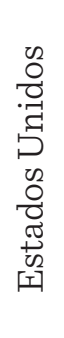 } & Nevada & mayo de 2017 \\
\hline Isabel & 23 & & Nevada & mayo de 2017 \\
\hline Luciana & 25 & & Nevada & mayo de 2017 \\
\hline Orencia & 19 & & Nevada & mayo de 2017 \\
\hline Petra & 21 & & Nevada & mayo de 2017 \\
\hline Rafaela & 22 & & Nevada & mayo de 2017 \\
\hline Rosario & 23 & & Nevada & mayo de 2017 \\
\hline
\end{tabular}

Los nombres son pseudónimos. 
Tabla 2. Descripción de los hombres entrevistados.

Table 2. Description of interviewed men.

\begin{tabular}{|c|c|c|c|c|c|}
\hline Entrevistado & País de origen & $\begin{array}{c}\text { Edad } \\
\text { (años) }\end{array}$ & $\begin{array}{c}\text { Sitio de } \\
\text { entrevista }\end{array}$ & Fecha & Comentarios \\
\hline Santiago & Guatemala & 41 & Nevada & $\begin{array}{l}\text { enero de } \\
2015\end{array}$ & $\begin{array}{l}\text { Trabajaba en un bar y en un mo- } \\
\text { tel de Nevada, como reclutador } \\
\text { de mujeres transportadas desde } \\
\text { Centroamérica por redes de tra- } \\
\text { ficantes que conducen mujeres } \\
\text { para el comercio sexual. }\end{array}$ \\
\hline Tomás & El Salvador & 48 & Nevada & $\begin{array}{c}\text { mayo } \\
\text { de } 2016\end{array}$ & $\begin{array}{l}\text { Trabajaba en un bar de Nevada, } \\
\text { como reclutador de mujeres la- } \\
\text { tinas, transportadas por redes de } \\
\text { traficantes que conducen muje- } \\
\text { res para el comercio sexual. }\end{array}$ \\
\hline Valerio & Guatemala & 44 & Nevada & $\begin{array}{c}\text { mayo } \\
\text { de } 2017\end{array}$ & $\begin{array}{l}\text { Trabajaba para una red de trafi- } \\
\text { cantes que suministraba mujeres } \\
\text { latinas a una agencia de Nevada. }\end{array}$ \\
\hline
\end{tabular}

Los nombres son pseudónimos.

Tabla 3. Características de las mujeres entrevistadas.

Table 3. Characteristics of the interviewed women.

\begin{tabular}{|l|c|c|c|c|c|c|}
\hline \multirow{2}{*}{ Característica } & \multicolumn{3}{c|}{ En tránsito por México } & \multicolumn{3}{c|}{ Residentes en Nevada } \\
\cline { 2 - 7 } & Media & Mín. & Máx. & Media & Mín. & Máx. \\
\hline Edad & 25.8 & 20 & 30 & 22.1 & 19 & 25 \\
\hline Edad cuando comenzaron a trabajar & 10.4 & 6 & 15 & 11.9 & 9 & 15 \\
\hline Años de educación & 1.6 & 0 & 6 & 5.2 & 0 & 8 \\
\hline Años de residencia en Nevada & 9.15 & 6.75 & 11.25 & 5.4 & 4 & 8 \\
\hline Entrevistadas n =12 & \multicolumn{3}{|c|}{5} & & 7 \\
\hline
\end{tabular}

años; 3 eran mexicanas, otras 3 procedían de Guatemala, y 1 era salvadoreña. Su edad estaba comprendida entre los 19 y los 25 años. Al igual que las mujeres en tránsito entrevistadas tuvieron que comenzar a trabajar a una temprana edad, para contribuir a la economía familiar; y el número promedio de años de educación era bajo, aunque superior al registrado por las que se encontraban en tránsito por México (Tabla 3). Por otra parte, en $\mathrm{Ne}-$ vada fueron entrevistados, entre 2015 y 2017 , 3 migrantes centroamericanos, conectados con redes de traficantes, que reclutaban mujeres de México y Centroamérica para ser introducidas en la prostitución en Nevada.
Debido a la naturaleza del objeto de estudio, es difícil saber si las redes estudiadas son las más activas, las más exitosas, las más visibles, o las que tienen más empleadas. Los participantes fueron contactados por los autores de este artículo a partir de personas que proporcionaban publicidad sobre servicios sexuales en Las Vegas Strip, Nevada. Se entrevistaron aquellas personas que accedieron a participar en este estudio. Por lo tanto, es posible que las redes estudiadas en esta investigación estén entre las que utilizan métodos menos violentos.

El trabajo de campo se realizó de acuerdo con los lineamientos propuestos por la Organiza- 
ción Mundial de la Salud, para investigar este tipo de población (Zimmerman y Watts, 2003). El diseño metodológico de esta investigación fue aprobado por el Comité de Ética de la Universidad Autónoma de Tamaulipas para estudios de Migración, desarrollo y derechos humanos (UAT, 2009). El instrumento aplicado fue una guía que incluía, tanto preguntas cerradas, que recogían las características socio-demográficas de los y las entrevistados/as, como preguntas abiertas, que exploraban cómo fueron reclutadas las mujeres, y los mecanismos de reclutamiento utilizados por los traficantes. A los y las participantes se les explicó el propósito de esta investigación y qué institución la realizaba, así como la naturaleza voluntaria de su participación en el estudio. Nadie recibió incentivos económicos por participar en este estudio, con objeto de no manipular su voluntad a través del otorgamiento de dicho incentivo. Se explicó a los y las participantes que los datos recogidos serían tratados con confidencialidad. Asimismo, para garantizar el anonimato de los discursos recabados, los y las participantes fueron instruidos para que no facilitasen su nombre, nombres de otras personas, ni datos específicos sobre la ubicación de lugares.

\section{RESULTADOS Y DISCUSIÓN}

\section{Introducción en la prostitución y recluta- miento por redes de traficantes}

Las entrevistadas tenían edades comprendidas entre 12 y 17 años cuando fueron prostituidas. Se estima que 10 de ellas fueron prostituidas en promedio 2.7 años en su lugar de origen, considerando la edad en que fueron prostituidas y la edad en que fueron trasladadas a Nevada. Tenían entre 12 y 19 años (solo 3 eran mayores de edad) cuando fueron transportadas por redes de traficantes hasta Estados Unidos (Tabla 4). Montiel-Torres (2018: 46) explicó que los tratantes sacan a las mujeres de su entorno familiar, social y afectivo, para trasladarlas a un lugar desconocido, con objeto de generar un estado de indefensión y dependencia, que facilita el inducirlas al comercio sexual. Una vez iniciadas en la prostitución, las jóvenes son vulnerables al tráfico sexual, ya que las redes de traficantes reclutan, en muchos casos, a jóvenes con experiencia en el comercio sexual en sus países de origen (IzcaraPalacios, 2017; 2018a). Esto hace que sea más fácil convencerlas para que emigren a Estados Unidos, con la promesa de salarios más elevados por llevar a cabo la misma actividad que realizan en su país (Izcara- Palacios y col., 2019). De acuerdo con el marco legal internacional, todas las mujeres entrevistadas fueron víctimas de trata, porque el hecho de ser menores de 18 años invalidaba su consentimiento. Aunque este punto de vista no es compartido por algunos académicos, que rechazan el discurso sobreprotector que equipara la sexualidad de los menores con el abuso sexual, incluso si fue sexo consensuado con un mayor de edad (Pheterson, 2004: 135; Agustín 2005: 110).

Las entrevistadas presentaron historial de abandono, abuso o violencia en su entorno familiar o personal; 3 de ellas quedaron embarazadas debido a múltiples violaciones por sus

Tabla 4. Edad cuando las entrevistadas fueron prostituidas.

Table 4. Age when the interviewed women were prostituted.

\begin{tabular}{|c|c|c|c|c|}
\hline \multicolumn{2}{|c|}{ Total (n=12) } & Media & Mínimo & Máximo \\
\cline { 2 - 5 } & Edad cuando fueron prostituidas por primera vez & 14.2 & 12 & 17 \\
\hline $\begin{array}{c}\text { Prostituidas en } \\
\text { sus países de } \\
\text { origen (n=10) }\end{array}$ & Edad cuando fueron prostituidas por primera vez & 14.3 & 12 & 17 \\
\hline
\end{tabular}


padrastros o por personas para quienes trabajaban; otras 3 quedaron embarazadas por una pareja violenta que las abandonó, y carecían de medios para mantener a sus hijos; en otros 3 casos, la madre murió o enfermó y ellas tuvieron que hacerse cargo de sus hermanos menores, y otra nunca conoció a su madre y vivió en la calle desde los 8 años. Ante ello, se podría deducir que, la situación de desamparo, aislamiento y precariedad económica de las entrevistadas fue aprovechada por proxenetas de sus países de origen para inducirlas a prostituirse. Por lo tanto, cuando años más tarde, un traficante les ofreció la oportunidad de ser conducidas a Nevada para trabajar en el comercio sexual, aceptaron su invitación. Asimismo, en Nevada recibieron documentación apócrifa que las acreditaba como mayores de edad. Como decía Rosario "en la tarjeta que me consiguieron para trabajar era ya mayor de edad".

Las 5 entrevistadas que fueron deportadas y se encontraban en tránsito por México traba- jaron en el comercio sexual durante más tiempo en Nevada que las 7 que residían en ese estado (9.2 años frente a 5.3 años de media). Las 12 mujeres entrevistadas habían trabajado en promedio, en años, el $12.2 \%$ del tiempo en México, el $13.8 \%$ en los países centroamericanos de origen y el $74 \%$ en Nevada. Las primeras, después de prostituirse en Nevada en diferentes establecimientos (casas de citas, antros, bares, clubs, hoteles, moteles, entre otros lugares) (Tabla 5), fueron deportadas a sus países de origen; 2 fueron detenidas tras una redada en el establecimiento donde se encontraban, 1 fue detenida en la calle, otra tras un incidente violento con su pareja, y 1 más fue delatada por una compañera de trabajo estadounidense.

De las 7 entrevistadas que residían en Nevada, 2 trabajaban como prostitutas en locales cerrados y 5 trabajaban para outcall referral agencies (agencias de entretenimiento adulto). Orencia y Gabriela fueron conducidas para trabajar en agencias, mientras que Rafae-

- Tabla 5. Número de años y lugar donde fueron prostituidas las entrevistadas.

Table 5. Number of years and place where the interviewees were prostituted.

\begin{tabular}{|c|c|c|c|c|}
\hline $\begin{array}{c}\text { Lugar donde fueron } \\
\text { prostituidas }\end{array}$ & Años & Deportadas (5) & $\begin{array}{l}\text { Residentes en } \\
\text { Nevada (y) }\end{array}$ & $\begin{array}{c}\text { Total } \\
(12)\end{array}$ \\
\hline \multirow{4}{*}{$\begin{array}{l}\text { País de origen } \\
\text { centroamericano }\end{array}$} & Media & 0.9 & 1.6 & 1.3 \\
\hline & Mínima & 0 & 0 & 0 \\
\hline & Máxima & 4 & 3 & 4 \\
\hline & Tiempo de prostitución (\%) & 7.9 & 20.0 & 13.8 \\
\hline \multirow{4}{*}{ México } & Media & 1.3 & 1 & 1.1 \\
\hline & Mínima & 0.3 & 0 & 0 \\
\hline & Máxima & 3.9 & 4 & 4 \\
\hline & Tiempo de prostitución (\%) & 11.7 & 12.7 & 12.2 \\
\hline \multirow{4}{*}{ Nevada } & Media & 9.2 & 5.3 & 6.9 \\
\hline & Mínima & 6.8 & 3 & 3 \\
\hline & Máxima & 11.3 & 8 & 11.3 \\
\hline & Tiempo de prostitución (\%) & 80.4 & 67.3 & 74.0 \\
\hline \multirow{4}{*}{ Total } & Media & 11.4 & 7.8 & 9.3 \\
\hline & Mínima & 7.6 & 5 & 5 \\
\hline & Máxima & 14.8 & 11 & 14.8 \\
\hline & Tiempo de prostitución (\%) & 100 & 100 & 100 \\
\hline
\end{tabular}


la, Luciana e Isabel, una vez pagada la deuda contraída con su patrón, pasaron a trabajar para una agencia. Algunas de las mujeres abandonan el comercio sexual, pero la falta de oportunidades laborales atractivas hace que regresen a esta actividad. Este es el caso de Luciana, que después de dejar el bar donde se prostituía buscó empleo en una actividad diferente. Tomó un curso para trabajar en un hotel como recamarera, pero después de trabajar unos meses decidió dejar este empleo y buscar trabajo en una agencia de entretenimiento adulto. Ella decía que el trabajo de recamarera, además de ser físicamente agotador, le reportaba un salario insuficiente para poder subsistir y enviar dinero a México para su hijo de 11 años.

"Trabajé un mes en un hotel, y pues, mejor me regresé a esto nuevamente, porque mira, no es el hotel el que te paga, sino es una empresa la que te contrata [...] No me pagaban por horas, sino por cuartos que yo hacía la limpieza, y primero estuve tomado un curso para aprender a hacer una habitación, y después del curso, que estuve pues como una semana, ya comencé a limpiar las habitaciones, y pues, nombre, no me gustó, porque ganaba poquillo" (Luciana).

\section{El abuso de una situación de vulnerabili- dad}

En los trabajos preparatorios para la elaboración del protocolo de Palermo, la presión ejercida por el comité de derechos humanos, donde participaban el movimiento pro-derechos de los trabajadores sexuales y activistas proderechos humanos, condujo a la introducción del concepto de consentimiento como elemento diferenciador entre la prostitución voluntaria y la trata (Doezema, 2010: 155; Andrijasevic, 2016: 61). Asimismo, desde el feminismo proprostitución se subraya que las mujeres que consienten en ser conducidas a otro país para trabajar en el comercio sexual no son víctimas de trata (Doezema, 2000; Pheterson, 2000; Blanchette y Da-Silva, 2012; Brents, 2016). Para Agustín (2007: 30-35), la mayor parte de las mujeres migrantes que trabajan en la industria del sexo no son víctimas, porque no fueron engañadas, el trabajo sexual les permite ganar dinero y ser independientes, y en ocasiones su estatus migratorio irregular las conduce a elegir situaciones de menos libertad.

Los reclutadores entrevistados en Nevada señalaban que las redes de traficantes siempre informaban a las mujeres sobre la actividad que realizarían en Nevada, de modo que eran ellas quienes tomaban la decisión de emigrar. Ellos afirmaban que las mujeres conducidas desde México y Centroamérica hasta Nevada sabían que trabajarían en la prostitución, y deseaban trabajar en esta actividad debido a los elevados salarios, y pagaban una elevada cantidad monetaria por ser conducidas hasta el lugar de destino. Santiago dijo: "aquí no se les engaña, ellas ya saben a lo que vienen y pagan para venir, $\mathrm{y}$ ya vienen con dirección y todo, y pues, aquí ya inmediatamente se habla con ellas para que sepan del trabajo". Asimismo, Valerio señaló: "las mujeres que traemos pagan por venir aquí a trabajar, para entretener a los hombres". Los entrevistados mencionaron que en Nevada, el número de mujeres que deseaban trabajar en el comercio sexual había crecido hasta sobrepasar la demanda. Por lo tanto, los patrones destinaban cada vez menos recursos al reclutamiento de mujeres. Rafaela coincidía con esta observación. Ella se quejaba del número tan elevado de jóvenes, tanto estadounidenses como extranjeras, que llegaban a Nevada para trabajar en la prostitución. Ella decía que cuando llegó a Nevada en 2013 los patrones reclutaban a jóvenes foráneas a través de redes de traficantes; pero en la actualidad no debían invertir dinero en procesos de reclutamiento, porque eran muchas las mujeres que llegaban a buscar empleo, tanto en establecimientos cerrados como en agencias de entretenimiento adulto.

"Vienen en vacaciones muchas chicas jóvenes de las universidades a trabajar, y pues, 
tú sabes, a ellas las buscan más, son colegialas y vienen con todo, se meten de bailarinas, y pues; antes pagaban por traerlas, como a mí; pero ya no, las mujeres vienen pagando por tener un trabajo aquí, y pues, aquí llega de todo, vienen de Colombia, de Centroamérica, de México, haitianas, nombre, de todas partes" (Rafaela).

Tomás, decía que debido a la falta de empleos, el número de mujeres que llegaban al bar donde trabajaba era superior a la demanda, de modo que, desde el año 2013, no habían contratado los servicios de redes de traficantes, porque las mujeres llegaban por su cuenta.

"Antes me encargaba de contactar con camaradas que traían mujeres para trabajar en el bar. Pero desde hace dos años las mujeres vienen solas, aquí llegan ellas mismas a buscar trabajo" (Tomás).

El relato de los reclutadores coincidía de modo parcial con el testimonio de las mujeres entrevistadas; 11 de las 12 mujeres entrevistadas dijeron que emigraron a Nevada de modo no coaccionado, y conocían que el trabajo que realizarían estaría relacionado con el comercio sexual. También, todas, excepto 2 , habían sido prostituidas antes de ser conducidas a Nevada. Esta situación pareciera indicar que la actividad delictiva realizada es solo tráfico de mujeres y no implica el delito de trata. Sin embargo, en el protocolo de Palermo, el consentimiento otorgado por una persona aparece anulado por la vulnerabilidad, un concepto subrayado por la Red Internacional de Derechos Humanos, de orientación neo-abolicionista (Doezema, 2010; Andrijasevic, 2016). Aunque, el protocolo de Palermo no define el término vulnerabilidad. La legislación estadounidense señala que, las mujeres y los niños son poblaciones vulnerables, pero no incluye el término vulnerabilidad en la definición de trata. Tampoco las legislaciones de Guatemala y Honduras definen el concepto de vulnerabilidad, y en El Salvador, la ley de trata ni siquiera enuncia este concepto. Como contraste, el artículo 4 (XVII), de la ley de trata de México, especifica 8 elementos y circunstancias definitorias del concepto "situación de vulnerabilidad”. El artículo 6 (16), de la Ley de trata de Nicaragua, especifica 15 elementos y circunstancias definitorias de este concepto (Izcara-Palacios, 2019b: 16-29). Igualmente, desde el feminismo abolicionista se subraya que toda forma de consentimiento de la mujer migrante en prostitución está viciado por una situación de vulnerabilidad (Orozco, 2015; Banyard, 2016; Coskun, 2016; Moran y Farley, 2019). Jeffreys (2011: 200) señaló que los defensores de la postura del trabajo sexual han tergiversado la definición de trata, al evitar la mención del concepto de "abuso de una posición de vulnerabilidad", ya que este término no implica un uso evidente de la fuerza.

En todos los casos estudiados, los traficantes y patrones abusaron de una situación de vulnerabilidad (las mujeres eran madres solteras, padecían una situación económica muy precaria, o querían escapar de un entorno familiar violento); 9 de las entrevistadas eran menores de 18 años cuando fueron transportadas a Nevada, y en 9 casos se produjo la concesión de pagos o beneficios para que las jóvenes emigrasen a Nevada. Estas redes reclutan muchas veces a menores de 18 años, con hijos, que tienen necesidades económicas muy apremiantes, porque son más fáciles de persuadir. Valerio decía que era muy fácil convencer a las jóvenes de México y Centroamérica para que emigrasen a Nevada a trabajar en la prostitución, debido a la alta tasa de embarazos de adolescentes que no encontraban empleo en sus lugares de origen, ni contaban con el apoyo de un varón.

"Aquí contacté con una agencia, y pues, sí quiere gente, sobre todo chava, chica, y pues, la verdad, ahora es más fácil conseguir, porque las chamacas quieren venirse para acá, porque quedan embarazadas muy chiquitas, y pues, no hay trabajo, y muchas veces se quedan solas porque los hombres, pues, no son como antes, ahora no se hacen cargo" (Valerio). 
La recepción de pagos o beneficios, para obtener el consentimiento de las menores de 18 años, es un mecanismo que las redes de traficantes utilizan con mucha frecuencia. Como señalaba Isabel: "el patrón pagó por mí, y aquí él me descontaba de mi pago cada semana". Sin embargo, las entrevistadas, lejos de sentirse engañadas, tendían a mostrar cierto agradecimiento, tanto hacia los traficantes que las ayudaron a llegar hasta Nevada, como hacia los patrones que les dieron empleo. Las entrevistadas subrayaban que nadie las engañó, que ellas tomaron la decisión de ir al norte. Además, no se arrepintieron de la decisión que tomaron, debido a las ganancias económicas que obtuvieron.

"Unos amigos me dijeron que se iban, que los llevaban, y me dijeron: vamos, y me fui. No me importó nada, me fui, ya tenía a mi hijo, lo dejé, no regresé a casa, no me obligaban, yo hice este trabajo porque así quise hacerlo" (Amalia).

"Quien me llevó hasta me pagó por adelantado para que me fuera, y me fui al norte. Me invitaron y me fui, porque allá me iba a ir mejor. Todo lo que me decían de por allá me hizo que sí me fuera, y qué bueno que me invitaron, porque allá sí me fue mejor, allá trabajé y me fue todo mejor" (Bruna).

"Tenía a un amigo que me ayudó a ir, ya antes me había invitado a ir, lo había conocido ahí donde trabajaba, en Honduras, él buscaba a mujeres que quisieran ir al norte y les ayudaba muy bien, y sí me ayudó a mí, poniéndome al pollero que me llevaría” (Eugenia).

Orencia, que fue conducida a Nevada a la edad de 14 años, ejemplifica la situación donde el consentimiento es obtenido por medio del abuso de una situación de vulnerabilidad y la recepción de beneficios. Ella no tenía ningún tipo de experiencia en el comercio sexual antes de ser conducida a Nevada. Tuvo que abandonar la escuela a la edad de 11 años para reemplazar a su madre, que era soltera y estaba embarazada, en un trabajo de mesera. Cuando su madre se reincorporó a este trabajo, ella encontró un empleo como sirvienta en la casa de un traficante, que conducía a jóvenes hasta Nevada para trabajar en la prostitución. Este traficante, le propuso conducirla de modo gratuito si ofrecía su virginidad al propietario de una agencia de entretenimiento adulto de Nevada. Ella relataba del siguiente modo cómo consintió en emigrar a Nevada.

"Trabajaba cuidando un niño, y pues, era la casa de un pollero, y pues, él me propuso el trabajo de venirme para acá [...] Pues, nombre, lo pensé como un mes, y pues, me animé porque, pues, mucha gente se viene y le va bien, y pues, me animé [...] No me cobró el pollero, me dijo que iba a pagar con mi virginidad, y pues, yo acepté porque, pues, era mucho dinero" (Orencia).

Las 11 entrevistadas que señalaban que nadie las engañó, dijeron que fueron invitadas a trabajar en Nevada por traficantes o madrotas que conocían y en quienes confiaban, y todo resultó como esperaban. Madrotas, para fines de este estudio, se define como mujeres dueñas de casas de citas, cantinas, casas de huéspedes, entre otros, cuya labor consiste en poner en contacto a las mujeres con los clientes, a cambio de un porcentaje del precio pagado por los últimos, que suele ascender al $50 \%$ (Izcara-Palacios, 2018a). Los traficantes que reclutaban jóvenes, para la industria del comercio sexual de Nevada eran personas que conocieron a través de familiares o en el lugar donde eran prostituidas en sus países de origen. La decisión de emigrar a Nevada no fue inmediata. En ocasiones meditaron durante meses las proposiciones que les hacían los traficantes, y finalmente accedieron a irse con ellos, porque los conocían y les tenían confianza. En la mayor parte de los casos no tendrían que pagar nada por desplazarse a un país donde ganarían salarios muy elevados, por realizar la misma actividad que hacían en sus países de origen. 
Expresiones como: "mi cuñado había trabajado en Estados Unidos y me presentó a un pollero, y él fue el que me trajo" (Isabel) o "mi mamá me animó a venirme, porque el pollero era amigo de mi abuelita, y pues, me dijo que confiara en el señor" (Orencia) describen los lazos existentes entre los traficantes y las jóvenes.

Las entrevistadas solían utilizar términos positivos para referirse a los traficantes que las condujeron hasta Estados Unidos, de quienes generalmente repetían que no abusaron de ellas, sino que las protegieron de los peligros del camino. Esto aparecía reflejado en expresiones como: "nos cuidó y nos llevó a donde nos dijo, no nos engañó ni nos mintió" (Adela); "el coyote no decía nada, solo nos llevaba, nos cuidaba" (Amalia); "me trató bien, me llevaba con mucho cuidado, cuidaba de mí, siempre me cuidaba" (Bruna) o "me daba un buen trato, me cuidaba mucho, porque el americano (el dueño de un bar de Nevada) me había encargado mucho" (Eugenia). Cabe mencionar que: la palabra "coyote", un término utilizado desde los años veinte, por los migrantes de la región migratoria tradicional del interior de México, constituye el vocablo más usado para designar a quienes facilitan la entrada clandestina a los Estados Unidos; también es el término más antiguo (Izcara-Palacios, 2015: 325).

En el caso de las redes mixtas, que transportan a hombres y mujeres, estas últimas, en ocasiones, buscan el apoyo de los traficantes para que las defiendan del acoso de los varones que viajan con ellas. Como señalaba Gabriela: "muchas veces, cuando llegaba la noche, ellos (los hombres) muchas veces te agarraban las piernas o te abrazaban esperando obtener algo; pero nunca me separaba del pollero que me trajo, él me respetaba, era un señor mayor, pero se portó muy bien conmigo". En pocos días llegaron hasta Nevada sin sufrir ningún percance, y allí les ofrecieron trabajar en sitios más lujosos que donde habían estado anteriormente, y su salario era más de 10 veces superior. Única- mente Isabel afirmó que, el lugar a donde la condujo el traficante, era más desagradable que la casa de citas donde fue prostituida en Guatemala desde los 13 años. Ella tuvo que trabajar más de un año en diferentes campos agrarios, donde no solo no había privacidad, sino que los jornaleros estaban desesperados por ser atendidos. Es por ello que ella, junto con otras compañeras, contactaron a través de internet con una agencia de Nevada, y dejaron de trabajar en los campos agrarios.

\section{El uso de métodos intimidatorios}

Los reclutadores entrevistados en Nevada se esforzaban por explicar que el negocio ilegal de la prostitución en Nevada se caracterizaba por la ausencia de métodos fraudulentos e intimidatorios, de modo que las mujeres reclutadas eran, generalmente, personas mayores de edad que habían decidido trabajar en la prostitución. Solo uno de los reclutadores mencionó que las redes de traficantes conducían a menores de 18 años; pero todos manifestaron que estas redes no engañaban a las mujeres. Sin embargo, a medida que avanzaba la entrevista, dejaron vislumbrar que la intimidación era un método utilizado de modo sistemático con las mujeres que no deseaban trabajar en la prostitución o querían abandonar el establecimiento donde se encontraban. Estas redes no recluyen a las mujeres en espacios fortificados donde son permanentemente vigiladas. Lograr la sumisión de las mujeres a través de la violencia física y del encerramiento no solo es costoso, sino también riesgoso, ya que podrían escapar y denunciarles (Izcara-Palacios, 2018b). Es más rentable y menos arriesgado ofrecer a las mujeres un salario atractivo, para así evitar tener que vigilarlas (Izcara-Palacios, 2020). Pero, para impedir que las mujeres dejen de trabajar en estos establecimientos o busquen empleo en otros lugares, donde las ofrezcan mayores incentivos económicos, estas redes deben ejercer algún tipo de intimidación. En muchos casos, la deportación o la amenaza de ser deportadas, constituye un mecanismo eficaz 
de control y sumisión de las mujeres migrantes. Aquéllas que no son suficientemente complacientes con los clientes, las que se rebelan contra sus patrones y les exigen mejores salarios, las que no son disciplinadas, o las que dejaron de ser rentables, debido a la edad o la pérdida de atractivo físico, pueden ser denunciadas para que las deporten las autoridades migratorias. Santiago explicaba cómo avisaron a las autoridades migratorias para que deportasen a una mujer que les resultaba problemática, porque no deseaba trabajar en el motel donde la prostituían.

"Ella ya no está con nosotros, y pues, aquí estuvo trabajando; pero ya nada más pues la usaron y otro colega le aventó la migra, porque, pues, no se vale que si ya se les dijo de qué se trata, aquí nadie engaña a nadie" (Santiago).

El uso de la intimidación aparecía más marcado en el discurso de los reclutadores que en el de las mujeres. Solo Engracia señaló que a la edad de 12 años fue engañada por un traficante que la condujo hasta Nevada. Ella decidió emigrar a Nevada porque la situación económica de su familia era desesperada. Engracia era la mayor de 8 hermanos, su madre tenía que cuidar de sus hermanos, su padre no tenía trabajo, y el traficante que llegó a su comunidad no llevaba a hombres, únicamente conducía a mujeres muy jóvenes hasta Nevada. Ella no entendía por qué a los hombres no les daban empleo en Estados Unidos, ni por qué querían mejor a las niñas que a las mujeres mayores. Ella se sentía afortunada porque la llevaban a Nevada, donde le dijeron que ganaría mucho dinero. Sin embargo, cuando llegó a Nevada, el trabajo que debía hacer no era lo que ella había imaginado.

"Estaba muy niña, con 12 años; pero así tuve que entrarle al trabajo, porque lo necesitaba y porque ya me tenían ahí donde iba a trabajar [...] Me llevaban a trabajar, me dijeron de trabajo; pero no me dijeron qué iba a ser el trabajo. Yo con las ganas y necesidad de trabajar dije que sí me iba a trabajar, y fui sin pensar que ése era el trabajo, y ya estando allá (en Nevada) tuve que hacerlo" (Engracia).

Los reclutadores señalaron que, en alguna ocasión, las mujeres a quienes condujeron hasta Nevada se arrepintieron de haberse comprometido a trabajar en la prostitución. Ellas deseaban devolver a los dueños de los bares, que pagaron su traslado, la suma que habían invertido en conducirlas hasta Estados Unidos, y trabajar en otra actividad. Sin embargo, los últimos pagan los elevados costos de conducir a una joven desde México y Centroamérica hasta Nevada, para que trabaje allí durante años, no para que les reembolse la suma invertida. Cuando una mujer acepta la oferta para ser conducida hasta Nevada, debe trabajar allí durante años, de modo que no le permiten buscar empleo en otro lugar. Adela explicaba que cuando fue reclutada en un prostíbulo de Tabasco (México), por una persona de Nevada, que la invitó a trabajar en el comercio sexual, tuvo que comprometerse a trabajar un mínimo de tres años para la persona que la reclutó.

"En ese lugar (un prostíbulo de Tabasco) conocí a un gringo, que me dijo que estaba muy bien, que él era de Nevada, de Estados Unidos, le gusté y me dijo que él venía a México a llevar mujeres que fueran a trabajar allá en lo mismo (la prostitución) y me invitó, no me llevó a la fuerza [...] Me dijo: 'si deseas ir, me hablas, y vemos; te puedo llevar, trabajas y me pagas por haber ido; pero si es que vas, tienes que trabajar para mí por tres años, después te vas a donde quieras" (Adela).

Amalia, que permaneció en Nevada desde noviembre de 2002, hasta abril de 2012, también explicaba que tuvo que permanecer durante 3 años en un bar. Cuando transcurrió el periodo abandonó ese lugar, para seguir trabajando en la prostitución en diferentes lugares cerrados. 
"En donde sí trabajé por 3 años fue en mi primer trabajo, era un bar; ya cuando me pude ir de ahí fui a trabajar a otro bar, trabajé por meses, de ahí me fui a un hotel, trabajé unos años; así estuve trabajando en diferentes lugares" (Amalia).

Igualmente, 3 de las entrevistadas, que residían en Nevada, después de pocos años de trabajo, pudieron abandonar a los patrones que las ayudaron a llegar a Estados Unidos. Rafaela, Luciana e Isabel, después de trabajar 2, 3 y 2 años, respectivamente, para los patrones que financiaron su traslado hasta $\mathrm{Ne}$ vada, abandonaron a su antiguo patrón para trabajar para una agencia de entretenimiento adulto, donde obtendrían mayores ingresos. Como señalaba Rafaela: "ahí (un motel) estuve trabajando 2 años, pagué lo que invirtieron por mí para traerme hasta aquí"; o como decía Luciana: "terminé de pagarle al pollero que me trajo y ahí conocí a mi novio, y pues, me ganché, y él me sacó de ahí (un bar)”.

Como contraste, 2 de las 5 entrevistadas que fueron deportadas, trabajaron para el mismo patrón durante una media de más de 9 años, ya que sus empleadores no les permitieron abandonar el lugar a donde fueron conducidas. Bruna, que permaneció en un bar de $\mathrm{Ne}-$ vada desde enero de 2005, hasta que fue deportada en febrero de 2014, señalaba: "al principio me dijeron 'no te puedes ir de este lugar, hay que trabajar, porque aquí te han traído', y ahí me quedé, ya no me cambié de trabajo, ahí el patrón me cuidaba bien, y ahí me acostumbré y trabajé todo el tiempo". Del mismo modo, Eugenia, que estuvo en un bar-club de un hotel desde agosto de 2006, hasta que fue deportada en noviembre de 2015, decía: "ahí tenía que estar, no podía cambiarme de trabajo; por eso nunca lo hice de irme de ahí donde había llegado a trabajar".

Los patrones que invierten dinero en conducir a mujeres indocumentadas hasta Nevada, no desean que estas abandonen los bares, clubs, casinos, hoteles, entre otros lugares, donde son prostituidas. Es por ello que les dicen, en tono amenazante, que no pueden abandonar los lugares de trabajo, porque contrajeron una deuda con ellos. Las mujeres conducidas por redes de traficantes hasta Nevada, no son vigiladas de cerca para evitar que puedan huir, aunque, sus patrones les infunden temor sobre los peligros de salir a la calle, ya que debido a su condición de migrantes indocumentadas pueden ser detenidas y deportadas. Sin embargo, algunas mujeres que se encuentran a disgusto en estos lugares se escapan. Como señalaba Engracia: "de la casa de citas me dijeron cuando llegué que no debía dejar de trabajar, que ahí tenía que estar trabajando porque me habían ayudado a estar ahí, y trabajar; pero cuando tuve problemas dejé el trabajo y me fui". Esta cita indica que aunque las mujeres padecían una relación contractual desventajosa, y sufrían explotación, tenían cierto margen de movilidad.

La literatura académica (Farley, 2007; Cacho, 2010; Farley, 2013; González-Báez, 2014; Montiel-Torres, 2015; Orozco, 2015; Banyard, 2016; Cobo, 2017; Shapiro y Hughes, 2017; Farley, 2018; Montiel-Torres, 2018; Moran y Farley, 2019) tiende a describir las redes de traficantes que conducen mujeres para el comercio sexual, como organizaciones manejadas por la delincuencia organizada transnacional, que utilizan la violencia, la coerción y el encerramiento, como instrumentos de operación estandarizados. En este estudio, los relatos de las entrevistadas no se adecuaban a la citada descripción. Sin embargo, es importante destacar que, la vulnerabilidad en la que viven algunas mujeres, es aprovechada por las redes de traficantes para transportarlas. La intimidación, con el recordatorio del pago del adeudo por cierto tiempo (años) o deportación (amenaza), cuando ya trabajan, son una constante. Por otro lado, la imposición de controles migratorios más estrictos, para combatir el tráfico de mujeres para el comercio sexual, si bien busca desalentar la actividad, también tiene un impacto contraproducente, ya que conduce a un incremento de las tarifas que pagan las mujeres, y eleva 
la deuda que contraen con las redes que las trasportan.

El modelo regulacionista de combate a la trata, tal como se implementa en países como Turquía, opera en perjuicio de la mujer migrante en prostitución, ya que se vale de su consentimiento para criminalizarla. Como ha señalado Coskun (2016), debido a que en Turquía se parte del axioma de que existe consentimiento, las mujeres migrantes en prostitución son detenidas y deportadas. Ya que no son catalogadas como víctimas, las mujeres son criminalizadas por entrar ilegalmente en el país, por ejercer la prostitución sin permiso y porque son focos potenciales de transmisión de enfermedades venéreas. Por una parte, anular toda forma de consentimiento de la mujer migrante en prostitución implica negar su capacidad de elegir. En el lenguaje kantiano, esto significa no reconocer la autonomía de su voluntad, que equivale a negar su racionalidad e inteligencia y, por lo tanto, su humanidad (Berlin, 2015: 109). Por otro lado, una aceptación irrestricta del concepto de consentimiento puede encubrir graves situaciones de vulnerabilidad.

\section{CONCLUSIONES}

Las redes de traficantes que conducen mujeres para el comercio sexual funcionan de modo similar a como lo hacen otras redes que conducen migrantes laborales. Los mecanismos de reclutamiento utilizados por las redes que operan en Nevada no suelen apoyarse en el uso de la violencia, sino en el abuso de una situación de vulnerabilidad. Estas redes reclutan frecuentemente a menores de 18 años con un pasado relacionado con la prostitución, que desean escapar de un entorno familiar violento $\mathrm{y} / \mathrm{o}$ tienen necesidades económicas apremiantes. Por lo tanto, no suelen valerse del engaño. El hecho de no usar estrategias de reclutamiento violentas, como el secuestro y el encierro, disminuye el riesgo de esta actividad, ya que las mujeres no les denuncian, porque no se consideran víctimas. Sin embargo, aunque, los relatos de los reclutadores, que se esforzaban por subrayar que ellos nunca recurrían al engaño, ni a la coacción, dejaban entrever, de modo más palpable que el discurso de las mujeres, que estas redes usan tácticas intimidatorias cuando las mujeres desean abandonar el comercio sexual. El tráfico internacional de mujeres para la prostitución es difícil de combatir, ya que existen mujeres de países pobres que buscan emigrar a países ricos para trabajar en el comercio sexual. La falta de oportunidades económicas para las mujeres en los países de emigración es lo que hace que, para algunas, el trabajo sexual en países ricos constituya una opción atractiva. Por lo tanto, la mejora de las oportunidades económicas para las mujeres en los países de origen, constituye una estrategia más efectiva de combate al tráfico sexual, que la prohibición del comercio sexual.

\section{AGRADECIMIENTO}

Este artículo es un producto del proyecto de investigación No CB-2013-01 220663 titulado "Trata y prostitución en México", financiado por la Secretaría de Educación Pública/Consejo Nacional de Ciencia y Tecnología (SEP/ CONACyT).

\section{REFERENCIAS}

Agustín, L. (2005). La industria del sexo, los migrantes y la familia europea. Cadernos Pagu. 25: 107-128.

Agustín, L. (2007). Sex at the margins. Migration, labour markets and the rescue industry. New York: Zed Books. 249 Pp.

Andrade-Rubio, K. L. (2016). Víctimas de trata: mujeres migrantes, trabajo agrario y acoso sexual en Tamaulipas. CienciaUAT.11(1):22-36.
Andrade-Rubio, K. L. e Izcara-Palacios, S. P. (2019). Las casas de seguridad de las redes de tráfico sexual en América del Norte. Oikos Polis, Revista Latinoamericana de Ciencias Económicas y Sociales.4(1):1-32.

Andrijasevic, R. (2016). Trafficking: Not a neutral concept. In S. Carrera and E. Guild (Eds.), Irregular migration, trafficking and smuggling of human beings. Policy dilemmas in the EU (pp. 58-63). Brussels: Centre for Euro 
pean Policy Studies.

Banyard, K. (2016). PIMP State. Sex, money and the future of equality. London: Faber \& Faber. $272 \mathrm{Pp}$.

Berlin, I. (2015). Las raíces del romanticismo. México D.F.: Taurus. $239 \mathrm{Pp}$.

Bingham, N. (1998). Nevada Sex Trade: A Gamble for the Workers. Yale Journal of Law \& Feminism. 10(1): 69-99.

Blanchette, T. G. and Da-Silva, A. P. (2012). On bullshit and the trafficking of women: moral entrepreneurs and the invention of trafficking of persons in Brazil. Dialectical Anthropology. 36(1-2): 107-125.

Blithe, S. J., Wolfe, A. W., and Mohr, B. (2019). Sex and Stigma: Stories of Everyday Life in Nevada's Legal Brothels. New York: NYU Press. 292 Pp.

Brents, B. G. (2016). Neoliberalism's market morality and heteroflexibility: Protectionist and free market discourses in debates for legal prostitution. Sexuality Research and Social Policy. 13(4): 402-416.

Brents, B. G. and Hausbeck, K. (2007). Marketing sex: US legal brothels and late capitalist consumption. Sexualities. 10(4): 425-439.

Brents, B. G., Jackson, C. A., and Hausbeck, K.(2010). The State of sex. Tourism, sex and sin in the New American heartland. New York: Routledge. 320 Pp.

Cacho, L. (2010). Esclavas del poder. Un viaje al corazón de la trata sexual de mujeres y niñas en el mundo. México D.F.: Grijalbo. 318 Pp.

Cobo, R. (2017). La prostitución en el corazón del capitalismo. Madrid: Catarata. 216 Pp.

Correa-Cabrera, G. and Sanders-Montandon, A. (2018). Reforming Mexico's anti-trafficking in persons legislation. Mexican Law Review. 11(1): 3-30.

Coskun, E. (2016). "Consent” Issue in Sex Trafficking and Evidence from Turkey. Social Politics: International Studies in Gender, State \& Society. 23(3): 437-458.

Doezema, J. (2000). Loose women or lost women? The re-emergence of the myth of white slavery in contemporary discourses of trafficking in women. Gender Issues. 18(1): 23-50.

Doezema, J. (2010). Sex slaves and discourse masters. The construction of trafficking. New York: Zed Books. 224 Pp.

Farley, M. (2007). Prostitution and trafficking in Nevada: Making the connections. San Francisco: Prostitution Research and Education. 328 Pp.

Farley, M. (2013). Prostitution, liberalism, and slavery, in Logos: A Journal of Modern Society \& Culture. [En línea]. Disponible en: http://logosjournal.com/2013/farley/ Google Scholar. Fecha de consulta: 22 de junio de 2019.

Farley, M. (2018). Risks of prostitution: When the per- son is the product. Journal of the Association for Consumer Research. 3(1): 97-108.

González-Báez, I. Y. (2014). El sistema proxeneta y las espirales de violencia y subordinación femenina en contextos de prostitución. [En línea]. Disponible en: http: //www.endslavery.va/content/endslavery/es/publications/ youth_symposium_2014/proxenetas.pdf. Fecha de consulta: 2 de febrero de 2020.

Hua, J. (2011). Trafficking women's human rights. Minneapolis: University of Minnesota Press. 184 Pp.

Izcara-Palacios, S. P. (2015). Coyotaje and Drugs: Two Different Businesses. Bulletin of Latin American Research. 34(3): 324-339.

Izcara-Palacios, S. P. (2017). El coyotaje visto desde la mirada de mujeres migrantes centroamericanas. Perfiles latinoamericanos. 25(49): 777-95.

Izcara-Palacios, S. P. (2018a). Prostitución de menores en locales registrados en México. Revista Internacional de Sociología. 76(1): 087.

Izcara-Palacios, S. P. (2018b). El déficit de denuncias de víctimas de prostitución forzada en México. Mexican Studies/Estudios Mexicanos. 34(2):191-21\%.

Izcara-Palacios, S. P. (2019a). Migración y trata en América del Norte. Revista de Estudios Sociales. (67): 87-100.

Izcara-Palacios, S. P. (2019b). Migración, prostitución y trata en México, Centroamérica y Estados Unidos. Ciudad de México: Fontamara. 150 Pp.

Izcara-Palacios, S. P. (2020). Trata, prostitución y capital erótico. Revista Internacional de Sociología.78(2).

Izcara-Palacios, S. P. y Andrade-Rubio, K. L. (2016). La deportación de mujeres centroamericanas víctimas de trata. Papeles de Población. 22(90):173-196.

Izcara-Palacios, S. P. y Andrade-Rubio, K. L. (2018). Centroamericanas menores de edad prostituidas en California. Nóesis: Revista de Ciencias Sociales y Humanidades. 27(53): 77-97.

Izcara-Palacios, S. P., Moral-de-la-Rubia, J. y Andrade-Rubio, K. L. (2019). Formas de inicio en la prostitución en menores latinoamericanas. Revista de Psicología y Ciencias del Comportamiento de la Unidad Académica de Ciencias Jurídicas y Sociales. 10(2). 44-66.

Jackson, C. A., Sahl, D., and Brents, B. G. (2018). Porn fans as sex tourists? Broadening meanings of sexual consumption among adult entertainment expo attendees in Las Vegas, Nevada. Porn Studies. 5(3): 241-256.

Jeffreys, S. (2010). "Brothels without Walls": the Escort Sector as a Problem for the Legalization of Prostitution. Social Politics: International Studies in Gender, State\& Society. 17(2):210-234. 
Jeffreys, S. (2011). La industria de la vagina. La economía política de la comercialización global del sexo. Buenos Aires: Paidós. 288 Pp.

Kant, I. (2017). Fundamentación de la metafísica de las costumbres. Ciudad de México: Austral. 159 Pp.

Lepp, A. (2002). Trafficking in women and the feminization of migration: The Canadian context. Canadian Woman Studies. 21(4): 90-99.

Luna-Alfaro, Á. C. (2016). Pensando el trabajo sexual desde una protagonista. Una visión a las intimidades de una cantina en el sur de Veracruz. Andamios. 13(30): 195210.

MacFarlane, R. T., Fuller, C., Wakefield, C., and Brents, B. G. (2017). Sex industry and sex workers in Nevada, in The Social Health of Nevada: Leading Indicators and Quality of Life in the Silver State. [En línea]. Disponible en: https://digitalscholarship.unlv.edu/social_health_nevada reports/57. Fecha de consulta: 18 de junio de 2019.

Montiel-Torres, O. (2015). La estructura básica de la explotación sexual. Propuesta de modelo teórico. Revista de Estudios en Antropología Sexual. 1(6): 83-101.

Montiel-Torres, O. (2018). El ciclo vital de las mujeres en situación de prostitución y el sistema proxeneta. Nueva Antropología.31(88): 31-51.

Moran, R. and Farley, M. (2019). Consent, coercion, and culpability: is prostitution stigmatized work or an exploitive and violent practice rooted in sex, race, and class inequality? in Archives of sexual be havior. [En línea]. Disponible en: https://doi.org/10.1007/s10508-018-13 71-8. Fecha de consulta: 12 de julio de 2019.

Orozco, R. (2015). Explotación sexual. Esclavitud como negocio familiar. México D.F.: L.D. Books. 174 Pp.

Pheterson, G. (2000). El prisma de la prostitución. Madrid: TALASA Ediciones, S. L.190 Pp.

Pheterson, G. (2004). Niños/as y prostitución: reflexiones críticas sobre la legislación y la edad. En R. Osborne (Ed.), Trabajadoras del sexo. Derechos, migraciones y tráfico en el siglo XXI (pp. 135-149). Barcelona: Ediciones Bellaterra.

Raphael, J. (2018). Decriminalization of Prostitution: The Soros Effect. Dignity: A Journal on Sexual Exploitation and Violence. 3(1): 1-32.

Rousseau, J. J. (1999). El contrato social. Madrid: Edimat Libros. 189 Pp.

Seals, M. (2015). Worker rights and health protection for prostitutes: A comparison of the Netherlands, Germany, and Nevada. Health Care for Women International. 36(7): 784-r96.

Shapiro, M. and Hughes, D. M. (2017). Decriminalized
Prostitution: Impunity for Violence and Exploitation. Wake Forest Law Review. 52: 533-560.

Skilbrei, M. L. (2019). Assessing the power of prostitution policies to shift markets, attitudes, and ideologies. Annual Review of Criminology. 2: 493-508.

Szasz, I. (2013). Sexualidad y cultura en México. Un ejercicio para la comprensión de los vínculos entre la cultura de género y la trata de personas con fines de explotación sexual. En R. Casillas (Ed.), Aspectos sociales y culturales de la trata de personas en México (pp. 41-132). México D.F.: INACIPE. 467 Pp.

UAT, Universidad Autónoma de Tamaulipas (2009). Protocolo del Comité de ética de la investigación. [En línea]. Disponible en: https://www.researchgate.net/publication/316554261_Protocolo_del_Comite_de_etica_de_la_ investigacion_del_CAC_UAT-CA-73. Fecha de consulta: 19 de junio de 2019.

Villacampa-Estiarte, C. (2012). Políticas de criminalización de la prostitución: análisis crítico de su fundamentación y resultados. Revista de Derecho Penal y Criminología. 7: 81-142.

Wagner, B. M., Whitmer, J. M., and Spivak, A. L. (2016). Experiences of youth in the sex trade in Las Vegas. New York: Center for Court Innovation. 48 Pp.

Walks, E. (2019). The Paradox of Policing as Protection: A Harm Reduction Approach to Prostitution Using Safe Injection Sites as a Guide. Duke Journal of Gender Law \& Policy. 26(2): 157-180.

Warren, K. B. (2012). Troubling the victim/trafficker dichotomy in efforts to combat human trafficking: the unintended consequences of moralizing labor migration. Indiana Journal of global legal Studies. 19(1): 105-120.

Zimmerman, C. and Watts, C. (2003). WHO ethical and safety recommendations for interviewing trafficked women. Geneva: World Health Organization. [En línea]. Disponible en: http://www.who.int/mip/2003/other_documents/ en/Ethical_Safety-GWH.pdf. Fecha de consulta: 29 de junio de 2019. 